Review

\title{
The Complex Nature of Adherence in the Management of HIV/AIDS as a Chronic Medical Condition
}

\author{
Adegoke O. Adefolalu ${ }^{1, *}$ and Zerish Z. Nkosi $^{1}$ \\ Department of Health Studies, University of South Africa, Pretoria, 0003, South Africa; \\ E-Mail: nkosizz@unisa.ac.za
}

* Author to whom correspondence should be addressed; E-Mail: gokeadef@hotmail.com; Tel: +27-12-380-1200; Fax: +27-86-764-9922.

Received: 15 July 2013; in revised form: 26 August 2013 / Accepted: 4 September 2013 / Published: 13 September 2013

\begin{abstract}
The introduction of antiretroviral therapy (ART) in the management of HIV infection has resulted in a significant reduction in the morbidity and mortality associated with the disease. The fact that a nearly perfect adherence is required in ART has remained a major challenge to people infected with HIV. This review underscores the impact of adherence to antiretroviral therapy and highlights recent advances in adherence monitoring and enhancement among people infected with HIV who are on lifelong antiretroviral therapy.
\end{abstract}

Keywords: adherence; antiretroviral therapy; HIV/AIDS

\section{Introduction}

Medical knowledge around HIV/AIDS has increased significantly over the years and good progress has been made in the treatment of HIV as a manageable life-threatening chronic condition using antiretroviral therapy (ART). The treatment of the disease extends beyond knowledge development among people infected with HIV; a partnership between them and healthcare providers is required, with the HIV-infected person assuming the major responsibility of self-care that will result in adherence and a good clinical outcome [1]. This is the reason why medication adherence is described as the extent to which the individual's behaviour corresponds to the prescribed medical advice of the health care provider [2]. This review provides an overview on adherence to ART among people infected with HIV by describing the pattern of adherence to treatment. It also includes the consequences of non-adherence to treatment, and the barriers and facilitators of ART adherence. 
Furthermore, the various modalities of adherence monitoring and assessment are described in detail, together with recent advances in strategies and tools used in enhancing adherence to antiretroviral therapy.

Medication adherence is a major challenge in chronic medical condition. Adherence levels change over time. Clinical experience and research indicate that adherence is a "moving target"; the longer a patient stays on treatment the poorer the adherence is likely to become [3,4]. Generally, adherence rates are higher among patients who are taking medications for acute medical conditions compared to those with chronic medical conditions [5]. In addition to this, adherence levels among patients with chronic diseases, no matter how impressive adherence is initially, have been reported to drop dramatically after six months [6]. HIV-infected persons have been shown to adhere better than the general population; the average adherence to chronic medications among the general population is $50 \%$ which is far below the self-report adherence rate of between $55-77 \%$ among patients on ART [7]. Despite this, non-adherence is common among patients on ART, which is estimated to be between $30-50 \%$, and more than $10 \%$ of patients usually miss one or more of their daily doses of antiretroviral drugs [8]. Adherence level among adolescents is usually lower than adults and elderly patients on ART [9,10]. In a study done in the USA among adolescents on antiretroviral drugs (ARVs), only 28\% took all their prescribed ARVs in the previous month [10]. Another cohort study in nine countries within the Southern Africa region also reported poor adherence among adolescents [11]. A survey done among HIV patients in India also revealed poor adherence among people who were aged below 40 years [3]. The explanatory factors responsible for this difference may be due to the fact that older individuals are more likely to have prior experience taking medication for age-related diseases and may have already become accustomed to such. Another reason may be related to the fact that lifestyle adjustments necessary for successful adherence are often less burdensome for adults compared to young patients [3].

Numerous interventional studies have been done in the past to address the problem of non-adherence among patients $[6,12,13]$. The unfortunate thing is that most of these interventions seem to have only modest impact [12-14]. The lack of appropriate theories to explain and predict non-adherence among patients on chronic medications may have a role to play in the slow progress made in developing interventions aimed at enhancing ART adherence [6,15].

\section{Adherence Required for Optimal Results in Antiretroviral Therapy}

The study of Paterson et al. [16] has been used widely in establishing the level required for optimal adherence necessary to maintain viral suppression (>95\%). In the study, the authors reported virologic failure (HIV RNA $>400$ copies/mL) in 22\% of patients with adherence level of $95 \%$ and above, $61 \%$ in patients with adherence level of $80-94.9 \%$ and $80 \%$ virologic failure in patients whose adherence level fell below 80\% [16]. Any adherence below 95\% in ART has been linked to treatment failure. The risk of developing resistance to ARV is also shown to be highest between 80-90\% level of adherence [17]. This is because the virus is not likely to develop resistance at low concentrations of the drugs but at a high concentration which is suboptimal for suppression of viral replication and the chances of viral resistance are very high $[17,18]$.

The earlier estimates of adherence levels necessary for viral suppression were done among patients receiving the unboosted protease inhibitors (PI) regimens which have been described as less potent than the non-nucleoside reverse transcriptase inhibitors (NNRTI) based-regimens [19,20]. Recent 
studies on adherence levels required to maintain good viral suppression with NNRTI-based regimens revealed that viral suppression was still achievable in patients with an adherence level below 80\% [21,22]. A South African study showed that there was improvement in patients' virologic outcomes as adherence to NNRTI-based regimens went beyond 50\% [21]. In addition, 73\% of the patients in the study achieved maximal viral suppression with adherence levels of 90-100\% [21]. This study shows that the NNRTI-based regimens may be an appropriate alternative to the PI-based regimens where adherence between $70-94 \%$ is anticipated, although the authors warned that adherence should be enhanced in any patients on ART irrespective of the patterns of adherence among populations groups [21]. Although the methods used in assessing adherence in some of these studies may not allow for the results to be generalized to other settings, what remains unclear is whether these results would be sustainable in the longer term as a very high adherence level is required indefinitely to maintain a continuous good clinical outcome in ART [16,22].

Several studies in sub-Saharan Africa have reported low adherence among patients on ART. In a sample of 109 patients on ART in Botswana, only 54\% of the patients were adherent by self-report, although this increased marginally to $56 \%$ when provider assessment was used [23]. In another study in Ethiopia conducted among 400 patients on ART [24], about 24\% were non-adherent when combined indicators of dose, time and dietary instructions were used. The percentage of non-adherent patients increased to $27 \%$ after reassessment three months later [24]. A Nigerian study that assessed ART adherence among patients on subsidized ART programme reported an alarming $75 \%$ of the study participants as non-adherent to their medications, patients in this study cited various reasons for their actions, ranging from ARVs side-effects and non-availability of ARVs to forgetfulness [25]. A more recent KwaZulu-Natal study among 735 HIV-infected persons found 30\% of the participants to be non-adherent to dose, schedule and dietary instructions [26]. Another South African study revealed that only $50 \%$ of patients on ART reported taking at least $95 \%$ of their prescribed ARVs [27]. A major review on studies conducted on ART adherence that involved 72 developed countries and 12 developing countries, five of which are African, estimated adherence level among people living with HIV/AIDS in Sub-Saharan Africa at 77\%, surprisingly higher than 55\% in North America. The authors stated further that non-adherence to ART in adult populations ranged between 33 and 88\%, depending on the measure of adherence employed [7]. These percentages are low considering that $95 \%$ adherence is required to minimize development of viral resistance and to achieve optimal benefit of ART [16].

\section{Consequences of Non-Adherence to Antiretroviral Therapy}

Adherence to ART is a good predictor of clinical outcome among patients on ART [16-18]. Adherence to combination ARV therapy has been shown to inhibit HIV replication which has resulted in the steady decline in HIV/AIDS related morbidity and mortality [28-30]. Lower levels of adherence have been demonstrated to achieve treatment goals in chronic medical conditions like hypertension and diabetes where moderate adherence has been considered adequate for treatment outcomes [14]. This is quite different in HIV/AIDS management where an adherence level greater than 95\% has been described as the requirement for maximal virological suppression especially ART regimens that contain the protease inhibitors [16,31]. Although recent studies indicate moderate adherence at $80-90 \%$ could suppress viral replication with the non-nucleotide reverse transcriptase inhibitors [21,22]; This finding 
has to be interpreted with caution since the risk of viral resistance and mortality is high at such adherence levels [32]. Unlike other chronic diseases where non-adherence is somehow tolerated, the high rate of viral replication and mutation in HIV means that high levels of adherence have to be maintained all times [18].

Non-adherence to ART results in inadequate suppression of viral replication in the body which allows the virus to continuously replicate and deplete the T-Helper cells; this destroys the immune system and allows the disease to progress at a faster rate [33]. Non-adherence is associated with repeated hospital admissions, development of opportunistic infections, poor quality of life, loss of productivity and premature mortality [32,34-36]. Development of resistance to ART is another consequence of non-adherence because the virus is prone to developing resistance to ARV when exposed to suboptimal concentrations of the drug [33,37]. These resistant strains could be transmitted to other persons thereby decreasing treatment options and worsening the HIV epidemic. Furthermore, various comparative studies among patients with poor adherence to ART showed that patients whose adherence levels were below $75 \%$ were three times more likely to die compared to those whose adherence levels were above $75 \%[21,38,39]$. Although, one has to bear in mind that many of the patients in these studies were initiated on ART with advanced stage of HIV/AIDS and with very low CD4 counts. The most important lesson from these studies is that poor adherence in advanced stages of the disease carries a high risk of mortality.

\section{Factors Influencing Adherence in Antiretroviral Therapy}

The management of HIV/AIDS with ART has resulted in significant clinical outcomes; but the complicated dosing requirements, ART adverse drug reactions and socio-economic factors often constitute a challenge to patients [24,40,41]. Although various studies in sub-Saharan Africa have shown that high levels of adherence, viral suppression and good clinical outcome are achievable in these resource-limited settings [7,11,32,42-45], it is very challenging to adhere to ART, and strict adherence is not common. [46] This does not mean adherence should be a barrier to ART initiation in developing countries with limited resources if there is adequate access to ART, proper patient education, social support, and issues around social barriers are well addressed [47]. The results obtained from some of the adherence studies in resource-limited settings should be interpreted with caution, as there is a possibility of the results skewing towards better adherence. The reason for this is because a large majority of participants in these studies received free ARVs, frees information and support from dedicated clinical staff, a beneficial experience that does not reflect the true situation of things in most resource-constraint settings [11,24,26,30,32,42,44]. Many studies have been done on factors associated with adherence in patients taking antiretroviral medication, while some have focused on socio-demographic characteristics like race, age, income and level of education; some focused have on health beliefs of patients; and others have concentrated on the interaction between patients and health care providers [24,27,30,40,41]. Although some of the factors identified in these studies are somehow predictive of adherence, none of them has been shown to be associated with adherence across studies. 


\subsection{Patient-Based Factors}

Socio-demographic factors such as age, gender, socio-economic status, level of education, income and ethnicity have been used in studies to understand their influence on adherence [48,49]. It has not been possible to absolutely predict adherence based on all these demographic characteristics as no consistent correlation has been found between demographic characteristics and patient adherence levels [3,48]. Psychosocial factors like drugs and alcohol use, social stability, depression and psychiatric illness have also been used in other studies to find out if there were correlation between any of them and adherence to ART [10,49-51]. What studies have found are barriers to adherence such as substance abuse, unstable housing, depression, mental illness, fear of disclosure of HIV status, decreased quality of life, work and family responsibility and past history of non-adherence [7,9,52]. Although socio-demographic factors have not been consistently correlated with adherence to ART in several studies, they can be used to identify particular populations that may benefit more extensively from targeted interventions that address specific barriers to adherence $[3,49]$. The importance of these findings in ART initiation is that denying an individual the benefit of ART based on the assumption that the person would not adhere due to his or her demographic characteristics is a futile exercise as no data are available to substantiate such action [4].

Other socioeconomic factors implicated as part of barriers to adherence to ART in a number of studies in Africa are the cost of ART, availability and accessibility to medications [23,43,53-56]. There are also some indirect costs associated with ART which influence adherence; these are the time taken off work, the time spent in hospital and inability to fend for one's family during bouts of opportunistic infections [43,57]. The access to drugs while away from home is another factor that has been identified which affects adherence among patients on ART [58,59]. Certain structural factors tend to influence adherence to ART, which are issues that are beyond the control of the patients [60]. Structural approach to adherence does not see adherence as a simple individual behaviour but as one that occurs within social and environmental contexts. In other words, it is difficult for some people to adhere to ART because the adherence barriers are not generated at an individual level [60]. Some of these are out-of-pocket cost of health services and medication, transportation, homelessness, lack of access to food and water and poor accessibility to health facilities. All these are structural barriers to adherence that have to be addressed at the societal level in order to improve adherence $[23,43,60]$. Reduction in these structural barriers to healthcare has been shown to improve patient's adherence levels in ART in some interventional studies to enhance adherence to ART [60].

\subsection{Treatment-Related Factors and Disease Characteristics}

ART is a complex treatment that is characterized by pill burden, dietary and fluid restrictions and timing of medication intake. The complexity of drug regimens is one of the causes of non-adherence among patients on chronic medication [3,52]. This has also been reported among HIV patients taking ARV; daily dosing regimens of three times or more are associated with non-adherence [60-62]. The pill burden is a major challenge since combination ART is used and it often contains 2-20 pills that have to be taken in a day together with the other factors to be considered like timing of dosages and food requirements [9]. The numerous and potentially debilitating side-effects contribute to irregular 
drug use and deliberate discontinuation of medication intake by some patients [23,63]. Regimens with significant side-effect profiles are usually associated with poor adherence [3,52]. Health care providers should consider the circumstances of patients while prescribing ART [64]; a potent combination therapy may not fit into a patient's daily schedule and may therefore affect the adherence to such medication. The rate of adherence to once-daily antiretroviral regimens has been found to be better than the rate of adherence to twice-daily regimens [65]. Regimen-based strategies that could improve long term adherence among patients include simplification of ART regimens which will assist in the long term adherence to treatment and maintaining efficacy of treatment $[3,9,51,61,64]$. The clinical outcomes achieved with the use of ART are linked to adherence levels [3]. Although the Swiss HIV Cohort Study revealed that long standing HIV disease was among the predictors of worsening adherence [52], prior opportunistic infections in an HIV patient before initiating ART have the potential of influencing adherence as the patient may perceive the disease to be severe enough to require good adherence to treatment in order to achieve the desired treatment outcome [3].

\subsection{Provider-Based Factors}

Providers' characteristics and clinical settings affect patients' adherence; overall patients' satisfaction with the level of care has been found to correlate with increased adherence [66]. The aspects of clinical setting that could positively influence adherence are a friendly and supportive environment, non-judgmental health care providers, convenient appointment schedule and confidentiality in service provision [67,68]. Long waiting times, poor staff attitudes, intermittent drug availability and other procedural barriers decrease patients' adherence to ART and also results in poor clinic attendance [53,54]. Continuous access to health care services and medications by patients also influences treatment adherence [53]. The patient-provider relationship is another factor that has been well researched in terms of adherence to ART; a good patient-provider relationship results in patient's trust and confidence in the provider which in turn influences good adherence [66,67].

The burden posed by adherence is not limited to the patient alone but extends to the health care provider; therefore, the relationship between the patient and the health provider should be that of a therapeutic alliance where both parties work toward a common goal of improving the health of the patient. This is why frequent change in health care providers is associated with poor adherence [52]. Discussing ART initiation with an informed patient is an effective strategy that enhances patient-provider interaction. It enables an alliance formation which allows the patient and the provider to define therapy goals, side-effects, medication management and adherence monitoring [64]. Social support and ability to disclose one's status are important issues to be considered when assessing treatment adherence irrespective of the context [32]. A study on adherence of patients to chronic medications recommends that therapeutic alliance between patients and providers should be strengthened, time should be devoted to address adherence among patients, and patients should be well assessed to identify barriers to treatment adherence [69].

\section{Monitoring and Assessing Antiretroviral Therapy Adherence}

Although adherence is one of the modifiable factors in ART, monitoring it is difficult among patients, since no standard method exists to monitor adherence in ART, and therefore, multiple 
approaches are often used $[7,11,68]$. Generally, ART adherence is calculated as the percentage of doses taken over those prescribed within a given period [2]. Specifically, medication adherence is defined as the percentage of prescribed medications taken in any form for a specified period; instructions adherence is defined as the percentage of medications for which the correct special instructions were followed at each prescribed dose [2,15]. Adequate monitoring of adherence is vital in preventing treatment failure and development of resistance to antiretroviral drugs [70]. Some of the commonest methods are self-report, electronic device monitoring, pills count, pharmacy refill tracking, biological markers, provider estimation and therapeutic drug monitoring.

\subsection{Self-Report of Adherence}

Self-report remains the main measure of adherence globally although other techniques have been used widely; it involves asking patients to report their adherence periodically [71]. Studies have found correlation with actual medication intake in research done on self-report as a method of adherence monitoring [44,71]. In the Ugandan study that used three-day self-report adherence, median adherence correlated with other measures of adherence [44]. The major drawback of this method is overestimation of adherence by patients due to desirability bias [72]. Adherence guidelines stipulate that accuracy of adherence self-report could be achieved if patients are approached in a nonjudgmental way during assessment [70].

\subsection{Pill Counts Method}

Pill count as a measure of adherence involves calculating the percentage of the number of pills prescribed and dispensed for the period between hospital appointments with the number of pills returned at the following appointment [42]. It is done during patients' visits to health facilities or unannounced at patients' home. This technique is cheap and correlates well with adherence measured using viral load [42], but its major shortcoming is manipulation of pills by the patient. Pill dumping prior to hospital visits has been documented which will result in overestimation of adherence for the patient. Unannounced pill count is more reliable but it has the tendency to affect the trust between the patient and health care provider which may eventually hinder adherence [22]. Though they may overestimate adherence; pill counts and self-report are still the mainstay of measuring adherence in resource-limited settings [73].

\subsection{Electronic Monitoring Devices (EMD)}

This involves an electronic chip that is implanted on the bottle lid that records the opening and closing of the bottle which contained the prescribed medicines, a computer program is later used to extract information from the lid and the data is then analyzed. This technique assumes that opening of the bottle by the patient coincides with actual intake of the drug. Although this method has shown some correlation with actual drug intake in some studies [16,51], others have described it as an expensive and non-reliable device [73]. A study in Malawi that compared methods of adherence monitoring found no correlation between electronic device monitoring, self-reporting and pill counts [73]. Electronic devices may underestimate or overestimate adherence since it is difficult to know if a 
patient opening a drug bottle is actually taking the tablets or just opening the bottle, therefore, actual medication intake by patients is not measured by EMD [74]. A study of electronic monitoring devices used among HIV patients on ART revealed that patients who rely on pill boxes are not likely to use EMD because pill boxes are the preferred tools for patients taking numerous medications everyday [74].

\subsection{Pharmacy Based-Records}

Pharmacy record is a simple and effective tool for monitoring adherence in ART and has been proved to be useful in adherence monitoring in a resource-limited setting [32,48]. This method employs pharmacy records to monitor adherence among patients when collecting their medication at the pharmacy. Patients collecting their medication regularly are said to be adherent by the pharmacy. Pharmacy records in resource limited settings are useful tools for measuring adherence; adherence measured by pharmacy records have been shown to correlate with CD4 count and weight gain in patients [72]. Adequate record keeping is necessary for pharmacy refill tracking information to be useful; the major setback is that the method only assumes that patients are adherent based on their empty pill boxes and the regular collection of their medication, it does not measure actual medication intake [32,48,72]. This method also requires patients to be collecting their medication at the same pharmacy for all refills which is not always possible [32,48,72].

\subsection{Biological Markers Monitoring}

Viral load level and CD4 counts are used as indicators for treatment outcomes and success. Viral load is regarded as the main indicator of the risk of therapeutic failure and can also be used in measuring adherence [42,75]. Low viral loads and increases in CD4 count are suggestive of good adherence although some patients may have a high viral load despite taking the ART regularly. CD4 is a good measure of adherence and correlated with weight gain, self-report and pharmacy records [72]. Viral load was also found to correlate with pill counts and good clinical outcomes in patients [42]. Pill counts may be a reliable and economical tool for monitoring adherence in resource-limited settings although viral load monitoring has been described as the preferred method [42]. The major drawback is cost and availability in resource limited settings [70].

\subsection{Provider Estimation Method}

In the provider estimation method of measuring adherence, the health care providers estimate a patient's adherence based on factors such as socio-demographic and economic factors. In this method, there is no correlation with actual medication intake; studies have shown that health care providers overestimate adherence to HIV treatment when factors such as demographic characteristics are used to predict adherence because it is very difficult to predict adherence based on demography [16]. Furthermore, consistent clinic attendance does not usually correlate with other measures of adherence among patients on ART [72]. 


\subsection{Therapeutic Drug Monitoring}

Therapeutic drug monitoring (TDM) involves measuring the levels of the drug in the blood stream of the HIV patient. This method is not used routinely as most ARVs have short circulating times in the body, coupled with the fact that it is very expensive [71]. Currently, therapeutic drug monitoring is restricted for research purposes. A study that combined TDM and viral resistance monitoring to find causes of virologic failure in patients on ART revealed that TDM is necessary in patients who developed adverse reactions while on a ritonavir boosting regimen because of the risk of drug toxicity in these patients [29]. TDM assists clinicians in choosing the best regimens for their patients and could be used routinely in clinical practice [29]. A prospective cohort study in Cameroon to compare adherence of fixed-dose combination of nevirapine, stavudine and lamivudine using nevirapine plasma level monitoring and self-report by patients found that self-reported adherence was significantly higher than adherence measures by nevirapine level monitoring [71]. The authors concluded that nevirapine plasma concentration monitoring provides an accurate measurement of adherence compared to self-report, but cautioned that it is not feasible in most clinical settings especially in resource-limited areas due to cost [71].

\section{Strategies and Tools for Enhancing Adherence}

Adherence is very complex and unpredictable among patients on chronic medications; several methods of enhancing adherence exist in ART and are usually used in combination for better results. The interventions used in enhancing adherence among patients are aimed at addressing potential barriers to adherence.

\subsection{Antiretroviral Therapy Strategies}

This entails simplifying regimen characteristics; simplification of dosing schedules, reduction of pill burden, and adjusting dietary restrictions to match patients' daily activities. In addition, identifying previous ART use and pre-existing medical conditions that could affect ART use and ensuring continuous provision of ART have also been reported to enhance adherence to ART [76]. Studies have demonstrated improved adherence with once or twice daily dosing of ART [77]. Many fixed-dose combinations (FDC) of ARVs are now available and have been shown to improve adherence in patients $[65,77,78]$. The buddy system has been widely used in resource-limited settings where relatives or friends agree to assist the patient in adhering to the medication [76]. Buddies remind patients to take their medication, encourage them and assist in keeping hospital appointments. This approach has led to improved ART adherence among some HIV-infected persons [76]. Social support not only assists the patient in adhering to ART but can also provide psychological support to the patient which helps them to cope with the disease [79].

\subsection{Education and Counselling}

This is usually the mainstay of ART programmes in any setting; Education and counselling empowers the patient to be part of the treatment process. Knowledge about the disease, its symptoms, treatment and side-effects of the medications are crucial information that has to be passed on to the 
patient [80]. During counselling, potential barriers to adherence are identified and addressed. Counselling assists the patient in developing positive beliefs and perception towards the disease [81]. It also helps in setting goals and increases the self-efficacy of the patient [82]. A randomized controlled trial in the USA to compare the effect of person-to-person contact and support adherence with medication alarm techniques revealed improved responses to therapy and good adherence among the group that received interpersonal adherence support [83]. This finding is consistent with the literature where repeated supportive adherence has been described as the most effective intervention because it provides human contact and support [68].

\subsection{Adherence Tools for Patients}

These tools are often combined with other behavioural interventions [84]. Pill boxes are containers used for storing the ARV for regular use as prescribed. They enable the patient to take the medication correctly. Electronic versions of pill boxes with reminders to the patient are also available. The major setback of this tool is the lack of confidentiality and privacy associated with it, since some patients do not want people around them to see their medications. Another setback is the task of filling out the boxes; uneducated patients might not be able to do this correctly. Pill charts involve visual display of the pills in terms of their colour, shape, name and dosage of the medication during counselling. This is very useful especially among the uneducated patients [84]. Electronic devices such as beepers, alarms and watches that remind patients to take their medication according to the prescribed schedule are also used to enhance adherence [70]. In addition, telephone calls and mobile-phone text messages have been shown to improve medication adherence in HIV infected individuals [85-88]. Electronic pagers linked to the internet may also be used to send reminders to patients to take their drugs. Although reminder tools have been reported by patients as one of the facilitators of adherence [85-88], the major disadvantage of this strategy is the lack of privacy associated with it. Another shortcoming is the cost required to set up this kind of service and the challenges associated with the patient having their mobile phones with them all the time [88]. Medication diaries are very useful in understanding the patterns of drug use by the patient and the reasons for not taking the medication regularly. Diaries are used by the patients to document the time and date of taking the medications and missed doses and the reasons for it. This tool may also be used to identify side-effects or other problems that the patient may encounter in the course of taking ART. Research has shown that some tools appear to be of more benefit to patients when they are combined with patient education or counselling. A large multicenter randomized trial in the USA revealed that reminder devices alone do not enhance adherence and suggests that they should be combined with counselling as part of comprehensive support for patients on ART [83].

\subsection{Directly Administered Antiretroviral Therapy (DAART)}

This technique has been used successfully in TB by asking patients to take medication under the supervision of adherence counsellors, trusted family members or community lay workers [89-91]. Directly administered ART can be clinic or home-based, once-daily, twice-weekly, or once-weekly [92]. The challenge in ART is different from TB as timing of medication intake varies across ARVs and the fact that the treatment is for life [93]. Although this approach has shown some benefit in improving 
adherence, research does not support routine use of directly administered ART [92-94]; Modified directly observed therapy (mDOT) has been suggested for use in ART and a home-based mDOT strategy has been used to improve adherence rates in resource-limited settings [92]. However, adherence to ART was only seen in the early weeks of a (mDOT) study in Kenya [94]. Despite its limited use, the benefits of mDOT in ART seen in early stages of treatment helped patients to develop good understanding of the treatment and develop good treatment taking behavior. It also assisted the patients in developing trusting relationships with the health care provider which subsequently improved their adherence to ART [92].

\subsection{Health System and Service Delivery Interventions}

These include interventions that target barriers to ART adherence at the level of health care delivery of HIV services such as food supplements, transportation to health facilities, staffing and integration of services. Research has demonstrated that ART adherence and retention in care are associated with interventions that offer food supplements, address transportation issues and integrated services. Family nutritional support for HIV positive patients on ART improves adherence leading to good clinical outcomes, and this could be integrated into ART adherence interventions as an effective and comprehensive community-based primary care [95]. In a South African study, nurse-initiated ART services have been shown to produce the same outcomes as ART services run by doctors, which indicates that where human resources are limited, nurses can provide ART care [96]. In addition, addressing the issue of transportation to the health facilities among HIV patients and integration of home-based care into ART services led to improved attendance in clinic appointments [97]. ART regimens need to be chosen taking into consideration the patient's working and family life, especially the cost implication of the drugs to ensure continuous supply so that adherence can be optimized [98].

\section{Conclusion}

Once initiated, antiretroviral therapy is a lifelong treatment; this has made adherence to ART one of the major challenges facing HIV/AIDS services following the rapid scale-up of ART to provide life-saving treatment to people infected with HIV. Non-adherence in ART is not limited to missing medication intake; it also includes other acts like not following instructions regarding dietary or fluid restrictions and not taking medication at the prescribed time. This underscores the complex nature of adherence in the management of HIV/AIDS as a chronic medical condition. Medication adherence is affected by patients' beliefs about disease origin and transmission, which often form the basis for stigmatization in HIV/AIDS. Adequate knowledge of the medication and understanding the need for strict adherence, self-efficacy, sense of self-worth, acceptance of HIV status, making use of reminder tools and social support are factors that influence adherence to ART in different settings [59]. Therefore, ART adherence needs to be tackled using a multi-disciplinary approach due to the difficultly in predicting which patients will adhere to treatment and those who will not; as a person's past adherence is the only predictor of future adherence [4]. 


\section{Conflicts of Interest}

The authors declare no conflict of interest.

\section{References}

1. Sanjobo, N.; Frich, J.C.; Fretheim, A. Barriers and facilitators to patients' adherence to antiretroviral treatment in Zambia: a qualitative study. J. Soc. Asp. H 2008, 5, 136-143.

2. Kenreigh, C.A.; Wagner, L.T. Medication adherence: A literature review. Medscape 12 October 2005.

3. Cauldbeck, M.; O’Connor, C.; O’Connor, M.; Saunders, J.; Rao, B.; Mallesh, V.; Kotechalappa, N.; Kumar, P.; Mamtha, G.; McGoldrick, C.; Laing, R.; Satish, S. Adherence to antiretroviral therapy among HIV patients in Bangalore, India. AIDS Res. Ther. 2009, 6, 7.

4. Ickovics, J.R.; Meade, C.S. Adherence to antiretroviral therapy among patients with HIV: A critical link between behavioural and biomedical sciences. J. Acq. Immun. Def. Synd. 2002, 31, S98-S102.

5. McDonald, H.P.; Garg, A.X.; Haynes, R.B. Interventions to enhance patient adherence to medication prescriptions. JAMA 2002, 288, 2868-2879.

6. Van Dulmen, S.; Sluijs, E.; van Dijk, L.; de Riddet, D.; Heerdink, R.; Bensing, J. Patient adherence to medical treatment: a review of reviews. BMC Health Serv. Res. 2007, 7, 55.

7. Mills, E.J.; Nachega, J.B.; Buchan, I.; Orbinski, J.; Attaran, A.; Singh, S.; Rachlis, B.; Wu, P.; Cooper, C.; Thabane, L.; et al. Adherence to antiretroviral therapy in sub-Saharan Africa and North America: A meta-analysis. JAMA 2006, 296, 679-690.

8. Chesney, M.A. Factors affecting adherence to antiretroviral therapy. Clin. Infect. Dis. 2000, 30, S171-S176.

9. Protopopescu, C.; Raffi, F.; Roux, P.; Reynes, J.; Dellamonica, P.; Spire, B.; Leport, C.; Carrieri, M.; and on behalf of the ANRS CO8 (APROCO-COPILOTE) Study Group. Factors associated with non-adherence to long-term highly active antiretroviral therapy: a 10-year follow-up analysis with correction for the bias induced by missing data. J. Antimicrob. Chemoth. 2009, 64, 599-606.

10. Murphy, D.A.; Sarr, M.; Durako, S.J.; Moscicki, A.; Wilson, C.M.; Muenz, L.R.; for the Adolescent Medicine HIV/AIDS Research Network. Barriers to HAART adherence among HIVinfected adolescents. Arch. Pediatr. Adolesc. Med. 2003, 157, 249-255.

11. Nachega, J.; Hislop, M.; Nguyen, H.; Dowdy, D.; Regensberg, L.; Chaisson, R.; Cotton, M.; Maartens, G. Antiretroviral therapy adherence, Virologic and immunologic outcomes in adolescents compared with adults in Southern Africa. J. Acq. Immun. Def. Synd. 2009, 51, 65-71.

12. Amico, K.R.; Harman, J.J.; Johnson, B.T. Efficacy of antiretroviral therapy adherence interventions a research synthesis of trials, 1996 to 2004. J. Acq. Immun. Def. Synd. 2006, 41, 285-297.

13. Munro, S.; Lewin, S.; Swart, T.; Volmink, J. A review of health behaviour theories: how useful are these for developing interventions to promote long-term medication adherence for TB and HIV/AIDS. BMC Public Health 2007, 7, 104.

14. Simoni, J.M.; Frick, P.A.; Pantalone, D.W.; Turner, B.J. Antiretroviral adherence interventions: A review of current literature and ongoing studies. Top. HIV Med. 2003, 11, 185-198. 
15. Kagee, A. Adherence to antiretroviral therapy in the context of the national roll-out in South Africa: Defining a research agenda for psychology. S Afr. J. Psychol. 2008, 38,413-428.

16. Paterson, D.L.; Swindells, S.; Mohr, J.; Brester, M.; Vergis, E.N.; Squier, C.; Wagener, M.M.; Singh, N. Adherence to protease inhibitor therapy and outcomes in patients with HIV infection. Ann. Intern. Med. 2000, 133, 21-30.

17. Bangsberg, D.R.; Moss, A.R.; Deeks, S.G. Paradoxes of adherence and drug resistance to HIV antiretroviral therapy. J. Antimicrob. Chemoth. 2004, 53, 696-699.

18. Clotet, B. Strategies for overcoming resistance in HIV-1 infected patients receiving HAART. AIDS Reviews 2004, 6,123-130.

19. Gazzard, B.G; on behalf of the BHIVA Treatment Guidelines Writing Group. British HIV Association guidelines for the treatment of HIV-1-infected adults with antiretroviral therapy 2008. HIV Medicine 2008, 9, 563-608.

20. Thompson, M.; Aberg, J.; Cahn, P.; Montaner, J.; Rizzardini, G.; Telenti, A.; Gatell, J.M.; Günthard, H.; Hammer, S.; Hirsch, M.; et al. Antiretroviral treatment of adult HIV infection: 2010 Recommendations of the International AIDS Society-USA Panel. JAMA 2010, 304, 321-333.

21. Nachega, J.B.; Hislop, M.; Dowdy, D.W.; Chaisson, R.E.; Regensberg, L.; Maartens, G. Adherence to non-nucleoside reverse transcriptase inhibitor-based HIV therapy and virologic outcomes. Ann. Intern. Med. 2007, 146, 564-573.

22. Bangsberg, D.R. Less than $95 \%$ adherence to nucleoside reverse transcriptase inhibitors therapy can lead to viral suppression. Clin. Infect. Dis. 2006, 43, 939-941.

23. Weiser, S.; Wolfe, W.; Bangsberg, D.; Thior, I.; Gilbert, P.; Makhema, J.; Kebaabetswe, P.; Dickenson, D.; Mompati, K.; Essex, M.; et al. Barriers to antiretroviral adherence for patients living with HIV infection and AIDS in Botswana. J. Acq. Immun. Def. Synd. 2003, 34, 281-288.

24. Amberbir, A.; Woldemichael, K.; Getachew, S.; Girma, B.; Deribe, K. Predictors of adherence to antiretroviral therapy among HIV-infected persons: a prospective study in southwest Ethiopia. BMC Public Health 2008, 8, 268.

25. Uzochukwu, B.S.C.; Onwujekwe, O.E.; Onoka, A.C.; Okoli, C.; Uguru, N.P.; Chukwuogo, O.I. Determinants of non-adherence to subsidized antiretroviral treatment in southeast Nigeria. Health Policy Plan. 2009, 24, 189-196.

26. Peltzer, K.; du Preez, N.; Ramlagan, S.; Anderson, J. Antiretroviral treatment adherence among HIV patients in KwaZulu-Natal, South Africa. BMC Public Health 2010, 10, 111.

27. Malangu, N.G. Self-reported adverse effects as barriers to adherence to antiretroviral therapy in HIV-infected patients in Pretoria. SA Fam. Pract. 2008, 50, 49.

28. Janssens, B.; Raleigh, B.; Soeung, S.; Akao, K.; Te, V.; Gupta, J.; Chhy Vun, M.; Ford, N.; Nouhin, J.; Nerrienet, E. Effectiveness of highly active antiretroviral therapy in HIV-positive children: Evaluation at 12 Months in a routine program in Cambodia. Pediatrics 2007, 120, e1134-e1140.

29. Duong, M.; Golzi, A.; Peytavin, G.; Piroth, L.; Froidure, M.; Grappin, M.; Buisson, M.; Kohli, E.; Chavanet, P.; Portier, H. Usefulness of therapeutic drug monitoring of antiretroviral in routine clinical practice. HIV Clin. Trials 2004, 5, 216-223. 
30. Ware, N.C.; Idoko, J.; Kaaya, S.; Biraro, I.A.; Wyatt, M.A.; Agbaji, O.; Chalamilla, G.; Bangsberg, D.R. Explaining adherence success in sub-Saharan Africa: an ethnographic study. PLOS Med. 2009, 6, e1000011.

31. Bangsberg, D.R.; Charlebois, E.D.; Grant, R.M.; Holodniy, M.; Deeks, S.G.; Perry, S.; Conroy, K.N.; Clark, R.; Guzman, D.; Zolopa, A.; et al. High levels of adherence do not prevent accumulation of drug resistance mutations. AIDS 2003, 17, 1925-1932.

32. Nachega, J.B.; Hislop, M.; Dowdy, D.W.; Lo, M.; Omer, S.B.; Regensberg, L.; Chaisson, R.E.; Maartens, G. Adherence to highly active antiretroviral therapy assessed by pharmacy claims predicts survival in HIV-infected South African adults. J. Acq. Immun. Def. Synd. 2006, 43, 78-84.

33. Rubbert, A.; Behrens, G.; Ostrowski, M. Ch 4-Pathogenesis of HIV-1 infection. In HIV Medicine; Hoffman, C., Rockstroh, J., Kamps, B., Eds.; Flying Publisher: Paris, France, 2007; pp. 59-81.

34. Graham, S.M.; Masese, L.; Gitau, R.; Jalalian-Lechak, Z.; Richardson, B.A.; Peshu, N.; Mandaliya, K.; Kiarie, J.N.; Jaoko, W.; Ndinya-Achola, J.; et al. Antiretroviral adherence and development of drug resistance are the strongest predictors of genital HIV-1 shedding among women initiating treatment. J. Infect. Dis. 2010, 201, 1538-1542.

35. Moore, D.M.; Hogg, R.S.; Yip, B.; Wood, E.; Tyndall, M.; Braitstein, P.; Montaner, J.S. Discordant immunologic and virologic responses to highly active antiretroviral therapy are associated with increased mortality and poor adherence to therapy. J. Acq. Immun. Def. Synd. 2005, 40, 288-293.

36. Wools-Kaloustian, K.; Kimayo, S.; Diero, L.; Siika, A.; Sidle, J.; Yiannoustsos, T.; Musick, B.; Einterz, R.; Fife, K.; Tierney, W. Viability and effectiveness of large-scale HIV treatment initiatives in Sub-Saharan Africa: experience from western Kenya. AIDS 2006, 20, 41-48.

37. Boyd, M.; Pett, S. HIV fusion inhibitors: a review. Aust. Prescr. 2008, 31, 66-69.

38. Hogg, R.S.; Heath, K.; Bangsberg, D.; Yip, B.; Press, N.; O'Shaughnessy, M.V.; Montaner, J.S. Intermittent use of triple-combination therapy is predictive of mortality at baseline and after 1 year of follow-up. AIDS 2002, 16, 1051-1058.

39. de Olalla, P.G.; Knobel, H.; Carmona, A.; Guelar, A.; Lopez-Colomes, J.L.; Cayla, J.A. Impact of adherence and highly active antiretroviral therapy on survival in HIV-infected patients. J. Acq. Immun. Def. Synd. 2002, 30, 105-110.

40. Dahab, M.; Charalambous, S.; Hamilton, R.; Fielding, K.; Kielmann, K.; Churchyard, G.J.; Grant, A.D. “That is why I stopped the ART”: patients' and providers' perspectives on barrier to and enablers of HIV treatment adherence in a South African workplace programme. BMC Public Health 2008, 8, 63.

41. Curioso, W.H.; Kepka, D.; Cabello, R.; Segura, P.; Kurth, A.E. Understanding the facilitators and barriers of antiretroviral adherence in Peru: a qualitative study. BMC Public Health 2010, 10, 13.

42. San Lio, M.M.; Carbini, R.; Germano, P.; Guidotti, G.; Mancinelli, S.; Magid, N.A.; Narciso, P.; Palombi, L.; Renzi, E.; Zimba, I.; et al. Evaluation of adherence to highly active antiretroviral therapy with use of pills counts and viral load measurement in the drug resources enhancement against AIDS and malnutrition program in Mozambique. Clin. Infect. Dis. 2008, 46, 1609-1616. 
43. Hardon, A.P.; Akurut, D.; Comoro, C.; Ekezie, C.; Irunde, H.F.; Gerrits, T.; Kglatwane, J.; Kinsman, J.; Kwasa, R.; Maridadi, J.; et al. Hunger, Waiting time and transport costs: Time to confront challenges to ART adherence in Africa. AIDS Care 2007, 19, 658-665.

44. Oyugi, J.H.; Byakika-Tusiime, J.; Charlebois, E.D.; Kityo, C.; Mugerwa, R.; Mugyenyi, P.; Bangsberg, D.R. Multiple validated measures of adherence indicate high levels of adherence to generic HIV antiretroviral therapy in a resource-limited setting. J. Acq. Immun. Def. Synd. 2004, 36, 1100-1102.

45. Fox, M.P.; Rosen, S. Patient retention in antiretroviral therapy programs up to three years on treatment in sub-Saharan Africa, 2007-2009: Systematic review. Trop. Med. Int. Health 2010, 15, $1-15$.

46. Montessori, V.; Press, N.; Harris, M.; Akagi, L.; Montaner, J. Adverse effects of antiretroviral therapy for HIV infection. Can. Med. Assoc. J. 2004, 170, 229-238.

47. Orrell, C.; Bangsberg, D.R.; Badri, M.; Wood, R. Adherence is not a barrier to successful antiretroviral therapy in South Africa. AIDS 2003, 17, 1369-1375.

48. Rougemont, M.; Stoll, B.; Elia, N.; Ngang, P. Antiretroviral treatment adherence and its determinants in Sub-Saharan Africa: A prospective study at Yaoundé central hospital, Cameroon. AIDS Res. Ther. 2009, 6, 21.

49. Gordillo, V.; del Amo, J.; Soriano, V.; Gonzalez-Lahoz, J. Socio-demographic and psychological variables influencing adherence to antiretroviral therapy. AIDS 1999, 13, 1763-1769.

50. Berg, K.M.; Cooperman, N.A.; Newville, H.; Arnsten, J.H. Self-efficacy and depression as mediators of the relationship between pain and antiretroviral adherence. AIDS Care 2009, 21, 244-248.

51. Howard, A.A.; Armsten, J.H.; Yuntai, L.; Vlahov, D.; Rich, J.D.; Schumand, P.; Stonee, V.E.; Smith, D.K.; Schoenbaum, E.E. for HER study group. A prospective study of adherence and viral load in a large multi-centre cohort of HIV-infected women. AIDS 2002, 16, 2175-2182.

52. Glass, T.R.; Battegay, M.; Cavassini, M.; De Geest, S.; Furrer, H.; Vernazza, P.L.; Hirschel, B.; Bernasconi, E.; Rickenbach, M.; Günthard, H.F.; Bucher, H.C.; The Swiss HIV cohort study. Longitudinal analysis of patterns and predictors of changes in self-reported adherence to antiretroviral therapy: Swiss HIV Cohort Study. J. Acq. Immun. Def. Synd. 2010, 54, 197-203.

53. Hawkins, C.; Murphy, R. Adherence to antiretroviral therapy in resource-limited settings: everything matters. AIDS 2007, 21, 1041-1042.

54. Iliyasu, Z.; Kabir, M.; Abubakar, I.S.; Babashani, M.; Zubair, Z.A. Compliance to antiretroviral therapy among AIDS patients in Aminu Kano Teaching Hospital, Kano, Nigeria. Niger J. Med. 2005, 14, 290-294.

55. Mukhtar-Yola, M.; Adeleke, S.; Gwarzo, D.; Ladan, Z. Preliminary investigation of adherence to antiretroviral therapy among children in Aminu Kano Teaching Hospital, Nigeria. AJAR 2006, 5, 141-144.

56. Tuller, D.M.; Bangsberg, D.R.; Senkungu, J.; Ware, N.C.; Emenyonu, N.; Weiser, S.D. Transportation costs impede sustained adherence and access to HAART in a clinic population in Southwestern Uganda. AIDS Behav. 2009, 14, 778-784.

57. Castro, A. Adherence to antiretroviral therapy: merging the clinical and social course of AIDS. PLoS Med. 2005, 2, e338. 
58. Nachega, J.B.; Stein, D.M.; Lehman, D.A.; Hlatshwayo, D.; Mothopeng, R.; Chaisson, R.E.; Karstaedt, A.S. Adherence to antiretroviral therapy in HIV-infected adults in Soweto, South Africa. AIDS Res. Hum. Retroviruses 2004, 20, 1053-1056.

59. Garcia, R.; Badaro, R.; Netto, E.; Silva, M.; Amorin, F.; Ramos, A.; Vaida, F.; Brites, C.; Schooley, R. Cross-sectional study to evaluate factors associated with adherence to antiretroviral therapy by Brazilian HIV-infected patients. AIDS Res. Hum. Retroviruses 2006, 22, 1248-1252.

60. Mukherjee, J.S.; Ivers, L.; Leandre, F.; Farmer, P.; Behforouz, H. Antiretroviral therapy in resource-poor settings: Decreasing barriers to access and promoting adherence. J. Acq. Immun. Def. Synd. 2006, 43, S123-S126.

61. Frank, I. Once-daily HAART: toward a new treatment paradigm. J. Acq. Immun. Def. Synd. 2002, 31, S10-S15.

62. Roca, B.; Lapuebula, C.; Vidal-Tregedor, B. HAART with didanosine once versus twice daily: adherence and efficacy. Int. J. Infect. Dis. 2005, 9, 195-200.

63. Waters, L.; Nelson, M. Why do patients fail HIV therapy? Int. J. Clin. Pract. 2007, 61, 983-990.

64. Harries, A.D.; Zachariah, R.; Lawn, S.D.; Rosen, S. Strategies to improve patient retention on antiretroviral therapy in sub-Saharan Africa. Trop. Med. Int. Health 2010, 15, 70-75.

65. Parienti, J.J.; Bangsberg, D.R.; Verdon, R.; Gardner, E.M. Better adherence with once-daily antiretroviral regimens: A meta-analysis. Clin. Infect. Dis. 2009, 48, 484-488.

66. Gauchet, A.; Tarquinio, C.; Fischer, G. Psychosocial predictors of medication adherence among persons living with HIV. Int. J. Behav. Med. 2007, 3, 141-150.

67. Altice, F.L.; Mostashari, F.; Friedland, G.H. Trust and acceptance of and adherence to antiretroviral therapy. J. Acq. Immun. Def. Synd. 2001, 28, 47-58.

68. Simoni, J.M.; Pearson, C.R.; Pantalone, D.W.; Marks, G.; Crepaz, N. Efficacy of interventions in improving highly active antiretroviral therapy adherence and HIV-1 RNA viral load: A metaanalytic review of randomized controlled trials. J. Acq. Immun. Def. Synd. 2006, 43, S23-S35.

69. Julius, R.J.; Novitsky, M.A.; Dubin, W.R. Medication adherence: a review of the literature and implications for clinical practice. J. Psychiatr. Pract. 2009, 15, 34-44.

70. Colebunders, R.; Moses, K.R.; Laurence, J.; Shihab, H.M.; Semitala, F.; Lutwama, F.; BakeeraKitaka, S.; Lynen, L.; Spacek, L.; Reynolds, S.J.; et al. A new model to monitor the virological efficacy of antiretroviral treatment in resource poor countries. Lancet Infect. Dis. 2006, 6, 53-59.

71. Kouanfack, C.; Laurent, C.; Peytavin, G.; Ciaffi, L.; Ngolle, M.; Nkene, Y.M.; Essomba, C.; Calmy, A.; Mpoudi-Ngole, E.; Delaporte, E.; et al. the French national agency for research on AIDS 1274 study group. Adherence to antiretroviral therapy assessed by drug level monitoring and self-reports in Cameroon. J. Acq. Immun. Def. Synd. 2008, 48, 216-219.

72. Ross-Degnan, D.; Pierre-Jacques, M.; Zhang, F.; Tadeg, H.; Gitau, L.; Ntaganira, J.; Balikuddembe, R.; Chalker, J.; Wagner, A.K.; INRUD IAA7. Measuring adherence to antiretroviral treatment in resource poor settings: the clinical validity of key indicators. BMC Health Serv. Res. 2010, 10, 42.

73. Bell, D.J.; Kapitao, Y.; Sikwese, R.; van Oosterhout, J.J.; Lallo, D.G. Adherence to antiretroviral therapy in patients receiving free treatment from a government hospital in Blantyre, Malawi. J. Acq. Immun. Def. Synd. 2007, 45, 560-563. 
74. Bova, C.A.; Fennie, K.P.; Knafi, G.J.; Dieckhaus, K.D.; Watrous, E.; Williams, A.B. Use of electronic monitoring devices to measure antiretroviral adherence: practical considerations. AIDS Behav. 2005, 9, 103-110.

75. Wilson, D.; Keiluhu, A.K.; Kogrum, S.; Reid, T.; Seriratana, N.; Ford, N.; Kyawkyaw, M.; Talangsri, P.; Taochalee, N. HIV-1 viral load monitoring: an opportunity to reinforce treatment adherence in a resource-limited setting in Thailand. T Roy. Soc. Trop. Med. H 2009, 103, 601-606.

76. Birbeck , G.L.; Chomba, E.; Kvalsund, M.; Bradbury, R.; Mang’ombe, M.; Malama, K.; Kail, T.; Byers, P.A.; Organek, N. for the RAAZ Study Team. Antiretroviral adherence in rural Zambia: The first year of treatment availability. Am. J. Trop. Med. Hyg. 2009, 80, 669-674.

77. Kauf, T.L.; Davis, K.L.; Earnshaw, S.R.; Davis, E.A. Spillover adherence effects of fixed-dose combination HIV therapy. Patient Prefer. Adherence 2012, 6, 155-164.

78. Haberer, J.E.; Cook, A.; Walker, A.S.; Ngambi, M.; Ferrier, A.; Mulenga, V.; Kityo, C.; Thomason, M.; Kabamba, D.; Chintu, C.; et al. Excellent adherence to antiretroviral in HIV+ Zambian children is compromised by disrupted routine, HIV nondisclosure, and paradoxical income effects. PLoS ONE 2011, 6, e18505.

79. Nachega, J.B.; Knowlton, A.R.; Deluca, A.; Schoeman, J.H.; Watkinson, L.; Efron, A.; Chaisson, R.E.; Maartens, G. Treatment supporter to improve adherence to antiretroviral therapy in HIV-infected South African adults: a qualitative study. J. Acq. Immun. Def. 2006, 43, S127-S133.

80. Magadza, C.; Radloff, S.E.; Srinivas, S.C. The effect of an educational intervention on patients' knowledge about hypertension, beliefs about medicines, and adherence. Res. Social Adm. Pharm. 2009, 5, 363-375.

81. Barclay, T.R.; Hinkin, C.H.; Castellon, S.A.; Mason, K.I.; Reinhard, M.J.; Marion, S.D.; Levine, A.J.; Durvasula, R.S. Age-associated predictors of medication adherence in HIV-positive adults: health beliefs, self-efficacy and neurocognitive status. Health Psychol. 2007, 26, 40-49.

82. Johnson, M.O.; Charlebois, E.; Morin, S.F.; Remien, R.H.; Chesney, M.A.; The NIMH Healthy Living Project Team. Effects of a behavioural intervention on antiretroviral medication adherence among people living with HIV: The Healthy Living Project randomized controlled study. J. Acq. Immun. Def. Synd. 2007, 46, 574-580.

83. Mannheimer, S.B.; Morse, E.; Matts, J.P.; Andrews, L.; Child, C.; Schmetter, B.; Friedland, G.H. (For the Terry Beirn community programs for clinical research on AIDS). Sustained benefit from a long-term antiretroviral adherence intervention: Results of a large randomized clinical trial. J. Acq. Immun. Def. Synd. 2006, 43, S41-S47.

84. Saberi, P.; Johnson, M.O. Technology-based self-care methods of improving antiretroviral adherence: A systematic review. PLoS ONE 2011, 6, e27533.

85. Bärnighausen, T.; Chaiyachati, K.; Chimbindi, N.; Peoples, A.; Haberer, J.; Newell, M.L. Interventions to increase antiretroviral adherence in sub-Saharan Africa: a systematic review of evaluation studies. Lancet Infect. Dis. 2011, 11, 942-951.

86. Van Velthoven, M.H.; Brusamento, S.; Majeed, A.; Car, J. Scope and effectiveness of mobile phone messaging for HIV/AIDS care: a systematic review. Psychol. Health Med. 2013, 18, 182-202.

87. Rodrigues, R.; Shet, A.; Antony, J.; Sidney, K.; Arumugam, K.; Krishnamurthy, S.; D’Souza, G.; DeCosta, A. Supporting Adherence to Antiretroviral Therapy with Mobile Phone Reminders: Results from a Cohort in South India. PLoS ONE 2012, 7, e40723. 
88. Skinner, D.; Rivette, U.; Bloomberg, D. Evaluation of use of cellphones to aid compliance with drug therapy for HIV patients. AIDS Care 2007, 19, 605-607.

89. Clarke, M.; Dick, J.; Zwarenstein, M.; Lombard, C.J.; Diwan, V.K. Lay health worker intervention with choice of DOT superior to standard TB care for farm dwellers in South Africa: a cluster randomized control trial. Int. J. Tuber. Lung Dis. 2005, 9, 673-679.

90. Manders, A.J.; Banerjee, A.; van den Borne, H.W.; Harries, A.D.; Kok, G.J.; Salaniponi, F.M. Can guardians supervise TB treatment as well as health workers? A study on adherence during the intensive phase. Int. J. Tuber. Lung Dis. 2001, 9, 838-842.

91. Wilkinson, D.; Davies, G.R.; Connolly, C. Directly observed therapy for tuberculosis in rural South Africa, 1991 through 1994. Am. J. Public Health 1996, 86, 1094-1097.

92. Farmer, P.; Leandre, F.; Mukherjee, J.; Gupta, R.; Tarter, L.; Kim, J.Y. Community-based treatment of advance HIV disease: Introducing DOT-HAART (directly observed therapy with highly active antiretroviral therapy). Bull. World Health Organ. 2001, 79, 1145-1151.

93. Lucas, G.M.; Flexner, C.W.; Moore, R.D. Directly administered antiretroviral therapy in the treatment of HIV infection: benefit or burden? AIDS Patient Care STDS 2002, 16, 527-535.

94. Sarna, A.; Lutchers, S.; Giebel, S.; Chersich, M.F.; Munyao, P.; Kaai, S.; Mandaliya, K.N.; Shikely, K.S.; Temmerman, M.; Rutenberg, N. Short and long term efficacy of modified directly observed antiretroviral treatment Mombasa, Kenya: a randomized trial. J. Acq. Immun. Def. Synd. 2008, 48, 611-619.

95. Serrano, C.; Laporte, R.; Ide, M.; Nouhou, Y.; Truchis, P.; Rouveix, E.; Adamou, A.; Pauly, V.; Mattei, J.F.; Gastaut, J.A. Family nutritional support improves survival, immune restoration and adherence in HIV patients receiving ART in developing country. Asia Pac. J. Clin. Nutr. 2010, 19, 68-75.

96. Sanne, I.; Orrell, C.; Fox, M.P.; Conradie, F.; Ive, P.; Zeinecker, J.; Cornell, M.;. Heiberg, C.; Ingram, C.; Panchia, R.; et al. for CIPRA-SA Study Team. Nurse versus doctor management of HIV-infected patients receiving antiretroviral therapy (CIPRA-SA): a randomized non-inferiority trial. Lancet 2010, 376, 33-40.

97. Andersen, M.; Hockman, E.; Smereck, G.; Tinsley, J.; Milfort, D.; Wilcox, R.; Smith, T.; Connelly, C.; Adams, L.; Thomas, R. Retaining women in HIV medical care. J. Assoc. Nurse AIDS C 2007, 18, 33-41.

98. Volberding, P.A.; Deeks, S.G. Antiretroviral therapy and management of HIV infection. Lancet 2010, 376, 49-62.

(C) 2013 by the authors; licensee MDPI, Basel, Switzerland. This article is an open access article distributed under the terms and conditions of the Creative Commons Attribution license (http://creativecommons.org/licenses/by/3.0/). 